\title{
Advancing subsurface biosphere and paleoclimate research: ECORD-ICDP-DCO-J-DESC-MagellanPlus Workshop Series Program Report
}

\author{
H. J. Mills ${ }^{1}$, J. de Leeuw ${ }^{2}$, K.-U. Hinrichs ${ }^{3}$, F. Inagaki ${ }^{4}$, and J. Kallmeyer ${ }^{5}$ \\ ${ }^{1}$ Division of Natural Sciences, University of Houston Clear Lake, Houston, TX, USA \\ ${ }^{2}$ NIOZ Royal Netherlands Institute for Sea Research, the Netherlands \\ ${ }^{3}$ MARUM Center for Marine Environmental Sciences, University of Bremen, Bremen, Germany \\ ${ }^{4}$ Kochi Institute for Core Sample Research, Japan Agency for Marine-Earth Science and Technology \\ (JAMSTEC), Kochi, Japan \\ ${ }^{5}$ GFZ German Research Centre for Geosciences, Potsdam, Germany
}

Correspondence to: H. J. Mills (geobiolab@gmail.com)

Received: 2 March 2015 - Revised: 20 May 2015 - Accepted: 26 May 2015 - Published: 17 December 2015

\begin{abstract}
The proper pre-drilling preparation, on-site acquisition and post-drilling preservation of high-quality subsurface samples are crucial to ensure significant progress in the scientifically and societally important areas of subsurface biosphere and paleoclimate research. Two of the four research themes of IODP and ICDP and one of the four research areas of the Deep Carbon Observatory (DCO) focus on the subsurface biosphere. Increasing understanding of paleoclimate is a central goal of IODP and incorporated within the scope of the IMPRESS program, the successor of the IMAGES program. Therefore, the goal of our IODP-ICDP-DCO-JDESC-MagellanPlus-sponsored workshop was to help advance deep biosphere and paleoclimate research by identifying needed improvements in scientific drilling planning and available technology, sample collection and initial analysis, and long-term storage of subsurface samples and data. Success in these areas will (a) avoid biological and other contamination during drilling, sampling, storage and shipboard/shore-based experiments; (b) build a repository and database of high-quality subsurface samples for microbiological and paleoclimate research available for the scientific community world-wide over the next decades; and (c) standardize, as much as possible, microbiological and paleoclimate drilling, sampling and storage workflows to allow results and data to be comparable across both space and time. A result of this workshop is the development and suggested implementation of new advanced methods and technologies to collect high-quality samples and data for the deep biosphere and paleoclimate scientific communities to optimize expected substantial progress in these fields. The members of this workshop will enhance communication within the scientific drilling community by crafting a handbook focused on pre-drilling, drilling and post-drilling operations.
\end{abstract}

\section{Scientific rationale}

For nearly two decades, a multidisciplinary, international effort has been conducted to describe the living subsurface biosphere. Although most research was conducted in the marine systems with the support of the International Ocean Discovery Program (IODP) and its predecessors (DSDP, ODP), exploration of the terrestrial subsurface biosphere through the International Continental Scientific Drilling Pro- gram (ICDP) has substantially increased in recent years. Microbial communities have been characterized in numerous sediment types representing a multitude of geologic ages, geochemical conditions, and geophysical constraints (IODP - Parkes et al., 2005; Schippers et al., 2005; Morono et al., 2011; Mills et al., 2012a; ICDP - Heim, 2011; Colwell and D'Hondt, 2013; Lau et al., 2014). As IODP embarks on a new decade, the time is right to coalesce the strengths and address the weaknesses from previous studies to re-develop 
and implement a plan to improve and standardize subsurface biosphere exploration. Due to the more individual or small group nature of drilling projects within ICDP, the European Consortium for Ocean Research Drilling (ECORD) and the Deep Carbon Observatory (DCO), the need for standardized biological sampling, processing and analysis becomes even more important.

The demand for biological samples has and will continue to increase as a result of the larger commitment to biology in the DCO and the new science plans of both IODP (2011) and ICDP (2014). The biological scientific goals within these groups focus on (a) the origin, composition and global significance of subsurface communities, (b) the scarcity of nutrients and energy and the limits of life in the subsurface, (c) the impact of environmental change on subsurface ecosystems and biodiversity and (d) the impact of subsurface communities on paleo-environmental and paleoclimate proxies, minerals and hydrocarbon reservoirs. Without the ability to compare results of multiple expeditions and access to properly acquired samples, these goals cannot be achieved. The biosphere community is aware of this challenge and understands the need for standardizing sample collection procedures, initial analysis protocols and long-term storage techniques (Orcutt et al., 2013; Kieft et al., 2015). We recognize that education of the broader drilling community is also required as many efforts related to biological sampling and characterization are seen as disruptive to normal core flow and description.

A unique challenge for the deep biosphere community has been fully communicating our requirements and resources to the drilling community. Biologists understand the need for collaborative data sets that characterize the chemical, physical and geological parameters of the sample. In many ways the drilling community views the biologist as a sink for drilling data; we use the data others produce but contribute little information in return to overall sample analysis. However, biologists can be a valuable source of subsurface data when consulted during core description and preservation efforts. By including biologically relevant chemical, physical and geological parameters within standard data sets, biologists can determine active microbial processes that may have altered or continue to alter parameters measured by other groups. The presence of active biological processes within retrieved samples can also have detrimental effects on legacy cores (Mills et al., 2012b). It is time for other disciplines to engage with biologists and biogeochemists in discussions about the effects of a living biosphere on the subsurface environment. For example, the application of proxies in paleoclimatology has become crucially important to reconstruct paleo-environmental settings and paleoclimate change. Since these proxies are based on organic compounds (biomarkers) and abundances and/or speciation of inorganic constituents, including stable isotope compositions, we must understand how these geochemical entities are affected by the microbial communities within the subsurface biosphere.
To further advance biosphere exploration in the scientific drilling community, a multidisciplinary team, including a molecular biologist, an ecologist, geochemists, and geologists, from nine countries assembled in Seoul, South Korea. The group represented leaders in the field of subsurface biosphere exploration in both the marine and terrestrial systems and possessed knowledge of prior similar workshops and initiatives to standardize subsurface biosphere techniques. The motivation for this workshop emerged during presentations and discussions at the Chikyu +10 meeting (Tokyo, 21-23 April 2013) and represents a successful extension from that meeting. Initial plans for this workshop exclusively focused on IODP-related research, but during the ICDP Science Conference in Potsdam, Germany (11-14 November 2013), it became clear that subsurface biosphere exploration faces the same challenges in both the marine and terrestrial realm. Therefore, the conveners broadened the workshop to cover additional programs including ICDP, DCO, and IMPRESS. Together these groups, and new ones to follow, have the potential to make a significant impact in subsurface biosphere research, the fastest developing field within the drilling community. Results from this workshop will be crucial in the development of the procedures required to accomplish the goals set forth in the new science plans and promoted through new initiatives.

One of our main goals in organizing this workshop was to advance the efforts of previous workshops. The 7th IODP Scientific Technology Panel that met on 28-30 July 2008 in Edmonton, Canada, expressed the need to standardize the biological sampling process and improve mechanisms for completing biological research within IODP. While procedural and policy changes have been implemented as a result of this 2008 meeting, several key challenges remained insufficiently addressed, including standard sub-sampling techniques for frozen samples, time- and redox-sensitive measurements, and submission of microbiology data and samples. ICDP first addressed subsurface biosphere research at their 2005 Science conference (Horsfield et al., 2007). In Potsdam 2009, an international workshop on the integration of deep biosphere research into ICDP listed potential drilling targets as well as technical and logistical prerequisites (Mangelsdorf and Kallmeyer, 2010). The growing strengths of DCO and IMPRESS provide additional momentum to subsurface biosphere exploration while presenting new and diverse challenges that must also be considered. Incorporating individuals present at these and other past meetings as well as those active within these different research communities was viewed as vital to the success of our workshop. In addition, individuals knowledgeable of new techniques and technologies were invited to address needs realized since the conclusion of the previous meetings. An open format for the workshop provided time for these groups to address past, present and future needs of the growing subsurface biosphere community. 


\section{The workshop}

The Advancing Subsurface Biosphere and Paleoclimate Research workshop took place in Seoul, South Korea, on 2123 August 2014, directly before the International Society for Microbial Ecology (ISME) meeting, also in Seoul. Twentyeight junior and senior scientists with experience in the geomicrobiological and biogeochemical components of the IODP, ICDP, DCO and IMPRESS (the successor of IMAGES) programs representative of the global community of subsurface microbiology, participated in this workshop and, for the first time, were able to speak with one solid voice. The idea for this workshop was developed during the Chikyu +10 workshop in April 2013, but had roots in many workshops, meetings and discussions over the last several years. Since the Chikyu +10 workshop, the idea to have a community discussion on standard protocols for microbiological drilling, sample handling and long-term sample storage developed rapidly with the interest and support of ICDP and DCO. Timing was important for these discussions since IODP, ICDP and DCO are at the beginning of new 10- and 5-year science plans with large geomicrobiological and paleoclimate components.

The overall aim of our workshop was to develop shared sampling and long-term storage strategies partly based on already existing white and scientific papers and to implement these strategies through standardized protocols for all drilling platforms, i.e., "traditional drilling" with the JOIDES Resolution, Chikyu and MSP/ICDP platforms. A decision was made during the workshop planning to expand the goal for standardization to much less expensive seabed drilling and long piston core operations from additional research vessels.

Initial workshop discussions were dedicated to providing background information on the current state of deep life research and proxy-based paleoclimatology within long-term scientific plans for IODP, ICDP, DCO and IMPRESS. In addition, presentations on subsurface microbiology and proxybased paleoclimatology highlighted the benefits of conducting geomicrobiological and paleoclimate research by acquiring high-quality microbiological samples, even when the expedition may focus on other scientific disciplines. At the end of day 1 and the start of day 2, most participants gave short $10-15$ min talks describing their specific research activities. Presentations emphasized their needs and experience with key aspects of pre-drilling, drilling, onboard sample handling, in-repository sample handling and long-term storage. Topics included sample frequency, contamination checks, core flow, geochemical measurements, cell enumeration, sample archives, data submission, staffing needs, education and collaborations with other scientific disciplines. Discussions that followed the presentations identified an apparent communication gap between the scientific drilling community and deep biosphere researchers that is inhibiting the progress of the entire community. The lack of communication was said to affect each stage of exploration from the pre- drilling planning stages through the drilling process to the post-drilling analysis. Misconceptions included the technical requirements for biological sampling, the frequency and targets for biological research, and sample preservation and storage. These issues are discussed below.

\section{Enhance communications and expectations}

Workshop participants suggested a three-level approach to improve the communication and expectation of biological research for upcoming drilling operations to help develop and implement a feasible set of standardized protocols for microbiological drilling, sample handling and long-term storage. Where possible, we took into account the diversity of drilling operations, i.e., "traditional" drilling, seabed drilling and long piston coring. While the expectations listed below are more specific to IODP, a similar level-based structure with program-specific expectation should be discussed and determined with these expectations acting as the catalyst for future discussion. The levels suggested are as follows.

\subsection{Level 1: expeditions with few to no geomicrobiological components}

Expectation: a technician trained in microbiology sampling will be on board. There will be low-frequency core sampling with no onboard contamination checks. Proposals listing this level for biology will not receive support from the biosphere community to improve rank when evaluated by the IODP proposal evaluation panel. Support will be provided to determine ways to enhance the biological components within the drilling plan and to demonstrate how these enhancements will help increase the scientific output of the expedition.

\subsection{Level 2: expeditions with a modest geomicrobiological component}

Expectation: at least one of the sailing scientists will be a microbiologist. A technician trained in microbiology sampling will also be on board. There will be more frequent core sampling with onboard contamination checks completed and limited supporting geochemistry compared to a Level 1 expedition. Proposals listed at this level will receive some support from the biosphere community to improve rank when evaluated by the IODP proposal evaluation panel. Support will be provided to determine ways to further enhance the biological components within the drilling plan and to demonstrate how these enhancements will help increase the scientific output of the expedition.

\subsection{Level 3: expeditions with a significant or predominant geomicrobiological component}

Expectation: two sailing scientists should be microbiologists. A technician trained in microbiology sampling will also be 
on board. There will be frequent core sampling with full onboard contamination checks, onboard cell counting, extended geochemical analysis and onboard CAS freezing facilities. For information on CAS freezing, please see Morono et al. (2015). Proposals listed at this level will receive full support from the biosphere community to improve rank when evaluated by the IODP proposal evaluation panel. Expedition advertisements and promotion within the biosphere community will ensure adequate pre-expedition planning, and onshore and offshore research, and post-expedition data and sample preservation will be made possible.

These levels are meant to promote proper communication of scientific expectations and requirements between expedition leaders and participants, an area that is currently plagued with misconceptions from both sides. Early communication during the proposal-writing phase will help guide sample requests and operational expectations of the chief scientists, biologists and drilling operators. The level approach was further refined through three subgroups organized based on an expedition planning and operation timeline. The groups were focused on predrilling and drilling, onboard sample processing and post-cruise legacy samples and data. Greater detail for these levels will be provided in the handbook being written by workshop participants. Details describing the handbook are provided below in Sect. 5 .

\section{Legal requirements for microbiological sampling within the economic exclusive zone (EEZ) through scientific drilling}

During the workshop, we discussed possible legal requirement issues for microbiological samples regarding the Nagoya Protocol on Access and Benefit-Sharing (ABS, http: //www.cbd.int/abs/). Briefly, the Nagoya Protocol on Access to Genetic Resources and the Fair and Equitable sharing of Benefits Arising from their Utilization to the Convention on Biological Diversity (CBD) is an international agreement at the government level, which aims at sharing the benefits arising from the utilization of genetic resources in a fair and equitable way. The Nagoya Protocol, created for greater legal certainty and transparency for both providers and users of genetic resources, was enacted on 12 October 2014, with the support of 57 countries. The Nagoya Protocol specified obligations to support compliance with the domestic legislation or regulatory requirements of the contracting party providing genetic resources, and contractual obligations reflected in mutually agreed terms. The contracting parties of the Nagoya Protocol are to take measures to ensure genetic resources utilized within their jurisdiction have been accessed in accordance with prior informed consent (PIC), and that mutually agreed terms (MAT) have been established, as required by another contracting party.

The United Nations Convention on the Law of the Sea (UNCLOS) establishes a plan of sovereignty over parts of the seas. The resources of the seas within state jurisdiction covered by UNCLOS are then accessed and shared according to the procedure established by the CBD and the Nagoya Protocol. The interaction of UNCLOS and the CBD means that the access regime of the CBD extends to all genetic resources (both terrestrial and marine) within the coastal state's jurisdiction. In this regard, the legal regulation is highly relevant to the deep biosphere-related sampling through IODP and other academic activities in the economic exclusive zone (EEZ). For example, during an IODP drilling project in the EEZ of a certain country, the samples used for biology are considered genetic resources belonging to that country, and should be taken following the regulations of the domestic law through the PIC and MAT. The complicacy of the Nagoya Protocol is that not all the party countries have already established the regulations and systems. Moreover, implementation of the Nagoya Protol in national legal systems has led to very diverse regulations that are different in each country. This might make microbiological sampling with IODP difficult when the drilling sites are located within the EEZ of a nation. ICDP drilling operations are affected as well.

Given the situation, the workshop participants recognized that it is important to share the knowledge among the IODP community and discuss possible issues that should be addressed properly before the drilling expedition at the IODP implementation organization level. Other organizations will have similar difficulties with the Nagoya Protocol and should also establish a working protocol to remain in compliance while not interfering with the biological exploration of the subsurface.

\section{DEEP BIO Handbook}

By the end of the workshop, a full handbook for microbiological and proxy-based paleoclimate drilling operations was determined to be both necessary and possible. The Deep Earth Exploration for Paleoclimate and Biosphere Investigation and Observation (DEEP BIO) Handbook is meant to promote proper communication of scientific expectations and requirements between expedition leaders and participants, an area that is currently plagued with misconceptions from both sides. As a result of discussions during the workshop, participants and selected experts were asked to assist in the writing of the handbook's four main sections: (1) Pre-drilling preparations and planning; (2) On-site operations; (3) Postdrilling processing and storage; and (4) Future Development (Table 1). Opportunities for community involvement in the writing process are discussed below.

Workshop participants, organized in breakout groups, identified multiple key points within each handbook section to help describe the needs of the biosphere community and where the biosphere community should be more proactive and involved. The early stages of proposal writing and planning were seen as a key time point for increased input from 
Table 1. Sections and topics to be included in the DEEP BIO Handbook.

\begin{tabular}{ll}
\hline Handbook section & Initial topics for discussion \\
\hline Pre-drilling preparations and planning & Proposal writing involvement \\
\hline & Sample request writing and expectation \\
& Procedure/methods/protocol preparations \\
\hline On-site operations & Contamination check technology and application \\
& Personnel needs \\
& Efficient and sterile sampling procedure \\
& On-site analysis in support of biological objectives \\
\hline Post-drilling processing and storage & Storage requirements to maximize sample viability \\
& Repository requests and sample availability \\
\hline Future development & Technologies for drilling, sampling and storage \\
& On-site technique developments \\
& Advances in data management \\
\hline
\end{tabular}

the biosphere community. Early activity in the proposal writing process will promote the inclusion of specific biological objectives within the main science plan and help alleviate misconceptions about the operational demand of biological sampling. Thus, sample requests, as well as drilling procedures, methods and protocols, should be discussed among all proponents in order to include a biological perspective of the overall goals of the drilling project. To accomplish biological inclusion in the proposal writing stage, monitoring of submitted proposals, involvement on planning committees and communication within the biosphere community are required.

The section describing on-site operations will focus on personnel needs, quality assurance and quality control protocols including the use of contamination checks, standardizing sampling procedures and requirements to increase efficiency and reduce core disruption, and on-site analysis protocols to maximize post-drilling research potential. Discussions were stimulated by recent work by Lloyd et al. (2013) that illustrated how different molecular methods used in multiple laboratories produced inconsistent results and thus suggested standardizing protocols to help link data sets. A standardized, high-throughput cell counting method, presented by Yuki Morono (Fig. 1), will be included in the handbook as one of the recommendations for on-site analysis (Morono et al., 2013). Additional methods will be considered with the understanding that the handbook should be updated as technologies advance.

Post-drilling preservation of both samples and data will be detailed in the third section. This will include recommendations as to how these resources can be made more accessible to a broader community. Additional mechanisms for sample storage and data archiving will be examined. Shifts in the metabolically active microbial community structure have been previously observed in cores under standard IODP storage conditions, suggesting alterations of the physical and

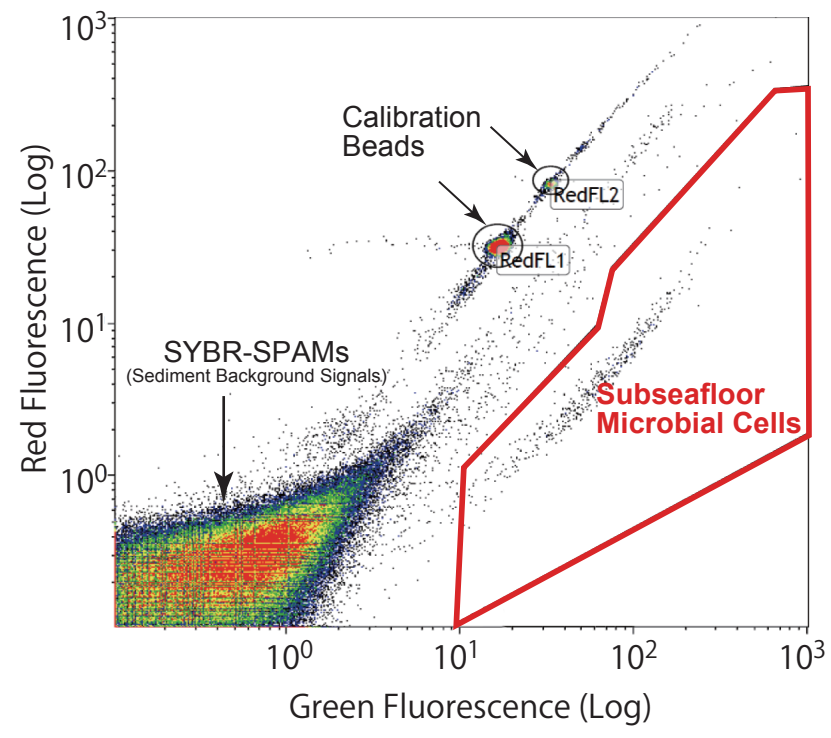

Figure 1. Standardization of cell counting techniques. The determination of cell abundance is a basic measurement common to most subsurface microbial community descriptions. An automated flow cytometer system can be used to determine cell abundances with high reproducibility and at a high sample rate (Morono et al., 2008). This method can be used repeatedly at multiple drill sites to reduce human errors and provide better connectivity between projects. (Figure is being used with permission from Yuki Morono; Morono et al., 2013.)

chemical characteristics (Mills et al., 2012b). The advanced preservation techniques and cryo-sampling procedures currently being used in the Kochi Core Repository DeepBIOS program led by Nan Xiao will described. Recommendations will be made to expand this program.

In the Future Development section, drilling technologies such as gel coring and large diameter side-wall coring will be 
highlighted to increase community discussion on sampling needs. This section will be used to help build new initiatives and discussions. It is our goal that new innovations will help advance biosphere exploration beyond the current challenges with the understanding that more challenges will be realized. Techniques and technologies in this section may be moved to other sections as they become more widely used.

The handbook will be initially tested during IODP expedition planning, drilling and post-expedition operations to determine feasibility and then used to train technicians and scientists. Protocols and procedures specific to different ocean and terrestrial drilling platforms, as well as within the repositories, will be included. Community involvement is welcome during the writing of this handbook. If you would like to contribute and be involved, please contact Heath Mills at geobiolab@gmail.com. The handbook will be designed to accommodate regular updates so that it may continue to guide subsurface exploration in the future.

The workshop successfully brought together an energetic and knowledgeable group that worked efficiently toward our goal of improving subsurface biosphere exploration. The key focal point discussed for moving forward was improving communication within the drilling community to improve overall understanding of the requirements and expectations for biosphere exploration. Deliverables from this workshop include an EOS newsletter article (Mills et al., 2014), this paper and a handbook to be written by workshop participants with community input.

Acknowledgements. We thank ECORD, ICDP, DCO, J-DESC and MagellanPlus for providing financial support to the workshop. We also thank N. Xiao for presenting the issue of microbiological sampling within the economic exclusive zone through scientific drilling. In addition, we would like to acknowledge the logistical support and overall assistance provided by J.-H. Lee.

Edited by: T. Wiersberg

Reviewed by: B. Horsfield and one anonymous referee

\section{References}

Colwell, F. S. and D'Hondt, S.: Nature and Exteme of the Deep Biosphere, in: Carbon in Earth. Mineralogical Society of America and Geochemical Society, Reviews in Mineralogy and Geochemistry, edited by: Hazen, R. H., Jones, A. P., and Baross, J. A., 75, 547-566, doi:10.2138/rmg.2013.75.17, 2013.

Heim, C.: Terrestrial deep biosphere, in: Encyclopedia of geobiology, edited by: Reitner, J. and Thiel, V., Dordrecht, The Netherlands, Springer ScienceCBusiness Media B.V., 871-876, 2011.

Horsfield, B., Kieft, T., Amann, H., Franks, S., Kallmeyer, S., Mangelsdorf, K., Parkes, J., Wagner, W., Wilkes, H., and Zink, K.-G.: The GeoBiosphere, edited by: Harms, U., Koeberl, C., and Zoback, M. D., Continental Scientific Drilling: A Decade of Progress and Challenges for the Future, Berlin-Heidelberg (Springer), 163-211, 2007.
ICDP: Unraveling the complexities of planet earth: science plan for 2014-2019, edited by: Horsfield, B., Knebel, C., Ludden, J., and Hyndman, R, International Continental Scientific Drilling Program, Potsdam, Germany, 2014.

IODP: Science plan for 2013-2023: Illuminating earth's past, present and future, Integrated Ocean Drilling Program Management International, Washington DC, 2011.

Kieft, T. L., Onstott, T. C., Ahonen, L., Aloisi, V., Colwell, F. S., Engelen, B., Fendrihan, S., Gaidos, E., Harms, U., Head, I., Kallmeyer, J., Kiel Reese, B., Lin, L.-H., Long, P. E., Moser, D. P., Mills, H., Sar, P., Schulze-Makuch, D., Stan-Lotter, H., Wagner, D., Wang, P.-L., Westall, F., and Wilkins, M. J.: Workshop to develop deep-life continental scientific drilling projects, Sci. Dril., 19, 43-53, doi:10.5194/sd-19-43-2015, 2015.

Lau, M. C. Y., Cameron, C., Magnabosco, C., Brown, C. T., Schilkey, F., Grim, S., Hendrickson, S., Pullin, M., Sherwood Lollar, B., van Heerden, E., Kieft, T. L., and Onstott, T. C.: Phylogeny and phylogeography of functional genes shared among seven terrestrial subsurface metagenomes reveal N-cycling and microbial evolutionary relationships, Front. Microbiol., 5, 531, doi:10.3389/fmicb.2014.00531, 2014.

Lloyd, K. G., May, M. K., Kevorkian, R. T., and Steen, A. D.: Metaanalysis of quantification methods shows that archaea and bacteria have similar abundances in the subseafloor, Appl. Environ. Microbiol., 79, 7790-7799, 2013.

Mangelsdorf, K. and Kallmeyer, J.: Integration of Deep Biosphere Research into the International Continental Scientific Drilling Program, Sci. Dril., 10, 46-55, doi:10.5194/sd-10-462010, 2010.

Mills, H. J., Reese, B. K., Shepard, A. K., Riedinger, N., Murano, Y., and Inagaki, F.: Characterization of the metabolically active bacterial populations in subseafloor Nankai Trough sediments above, within and below the sulfate-methane transition zone, Front. Microbiol., 3, 113, doi:10.3389/fmicb.2012.00113, 2012a.

Mills, H. J., Reese, B. K., and St. Peter, C.: Characterization of microbial population shifts during sample storage, Front. Microbiol., 3, 49, doi:10.3389/fmicb.2012.00049, 2012 b.

Mills, H. J., de Leeuw, J., Kinrichs, K. U., Inagaki, F., and Kallmeyer, J.: Advancing Sub-Surface Biosphere and Paleoclimate Research MagellanPlus Workshop - 21-23 August 2014, Seoul (South Korea), ECORD Newsletter, 23, p. 21, 2014.

Morono, Y., Terada, T., Masui, N., and Inagaki, F.: Improved and automated cell count system for rapid enumeration of microbial cells in the deep subsurface, Geochem. Cosmochim. Ac., 72, A651-A651, 2008.

Morono, Y., Terada, T., Nishizawa, M., Ito, M., Hillion, F., Takahata, N., Sano, Y., and Inagaki, F.: Carbon and nitrogen assimilation in deep subseafloor microbial cells, P. Natl. Acad. Sci. USA, 108, 18295-18300, 2011.

Morono, Y., Terada, T., Kallmeyer, J., and Inagaki, F.: An improved cell separation technique for marine subsurface sediments: applications for high-throughput analysis using flow cytometry and cell sorting, Environ. Microbiol., 15, 2841-2849, doi:10.1111/1462-2920.12153, 2013.

Morono, Y., Terada, T., Yamamoto, Y., Xiao, N., Hirose, T., Sugeno, M., Ohwada, N., and Inagaki, F.: Intact preservation of environmental samples by freezing under an alternating magnetic 
field, Environ. Microbio. Rep., 7, 243-251, doi:10.1111/17582229.12238, 2015.

Orcutt, B. N., LaRowe, D. E., Biddle, J. E., Colwell, F. S., Glazer, B. T., Reese, B. K., Kirkpatrick, J. B., Lapham, L. L., Mills, H. J., Sylvan, J. B., Wankel, S. D., and Wheat, C. G.: Microbial activity in the marine deep biosphere: progress and prospects, Front. Microbiol., 4, 1-14, doi:10.3389/fmicb.2013.00189, 2013.

Parkes, R. J., Webster, G., Cragg, B. A., Weightman, A. J., Newberry, C. J., Ferdelman, T. G., Kallmeyer, J., Jørgensen, B. B., Aiello, I. W., and Fry, J. C.: Deep sub-seafloor prokaryotes stimulated at interfaces over geologic time, Nature, 436, 390-394, 2005.
Schippers, A., Neretin, L. N., Kallmeyer, J., Ferdelman, T. G., Cragg, B. A., Parkes, R. J., and Jørgensen, B. B.: Prokaryotic cells of the deep sub-seafloor biosphere identified as living bacteria, Nature, 433, 861-864, 2005. 